\title{
Identifying developmental trajectories of body mass index in childhood using latent class growth (mixture) modelling: associations with dietary, sedentary and physical activity behaviors: a longitudinal study
}

Maaike Koning ${ }^{1 *}$, Trynke Hoekstra ${ }^{2,3}$, Elske de Jong ${ }^{1,4}$, Tommy L. S. Visscher $^{1}$, Jacob C. Seidell ${ }^{1,2}$ and Carry M. Renders ${ }^{1,2}$

\begin{abstract}
Background: To date, many epidemiologic studies examining associations between obesity and dietary and sedentary/physical activity behaviors have focused on assessing Body Mass Index (BMI) at one point in time. Recent developments in statistical techniques make it possible to study the potential heterogeneity in the development of $\mathrm{BMI}$ during childhood by identifying distinct subpopulations characterized by distinct developmental trajectories. Using Latent Class Growth (Mixture) Modelling (LCGMM) techniques we aimed to identify BMI trajectories in childhood and to examine associations between these distinct trajectories and dietary, sedentary and physical activity behaviors.

Methods: This longitudinal study explored BMI standard deviation score (SDS) trajectories in a sample of 613 children from 4 to 12 years of age. In 2006, 2009 and 2012 information on children's health related behaviors was obtained by parental questionnaires, and children's height and weight were measured. Associations with behaviors were investigated with logistic regression models.

Results: We identified two BMI SDS trajectories; a decreasing BMI SDS trajectory ( $n=416 ; 68 \%)$ and an increasing BMI SDS trajectory $(n=197 ; 32 \%)$. The increasing BMI SDS trajectory consisted of more participants of lower socio-economic status (SES) and of non-western ethnicity. Maternal overweight status was associated with being in the increasing BMI SDS trajectory at both baseline and follow-up six years later (2006: Odds Ratio (OR), 2.9; 95 \% confidence interval (Cl) 1.9 to 4.3; $2012 \mathrm{OR}, 1.8 ; 95 \%$ Cl 1.2 to 2.6). The increasing BMI SDS trajectory was associated with the following behaviors; drinking sugared drinks $>3$ glasses per day, participation in organized sports $<1 \mathrm{~h}$ per week, and TV viewing $>2 \mathrm{~h}$ per day, though participation in organized sports at follow-up was the only significant result. (Continued on next page)
\end{abstract}

\footnotetext{
* Correspondence: m.koning@windesheim.nl

${ }^{1}$ Research Centre Healthy Cities, Knowledge Centre for Health and Social work, Windesheim University of Applied Sciences, PO box 10090, 8000 GB Zwolle, The Netherlands

Full list of author information is available at the end of the article
} 
(Continued from previous page)

Conclusions: Our results indicate the importance of healthy lifestyle behaviors at a young age, and indicate that maternal $\mathrm{BMl}$ is a very important risk factor for the development of childhood overweight. Comprehension of heterogeneity in the development of BMI and associations with modifiable health related behaviors is interesting for prevention by targeting high risk behaviors in early childhood, especially in low SES children, children of non-western ethnicity and children whose mother is overweight.

Keywords: Developmental trajectory, Latent class growth (mixture) models, Longitudinal study, Childhood overweight, Health related behaviors, Body mass index

\section{Background}

In populations worldwide, the prevalence of childhood overweight and obesity has dramatically increased during the last decades [1-5]. This increase has also been observed in the Netherlands [6-8]. Compared to 1980, the prevalence of childhood overweight in 2009 has increased two to three fold and the prevalence of obesity increased four to sixfold [7]. In $200912.8 \%$ of the boys and $14.8 \%$ of the girls aged 2 to 21 years were classified as overweight and $1.8 \%$ of the boys and $2.2 \%$ of the girls were classified as obese.

Increasingly, children become overweight at a relatively young age, as shown in many countries in large surveys with data for children aged younger than 5 years separated by half a decade or more [5]. Being overweight and especially being obese as a child increases the risk of becoming an overweight adult [9-11], and has been associated with serious comorbidities in adulthood such as cardiovascular risk factors $[12,13]$. In the short term, obesity is associated with medical problems such as elevated blood pressure, type 2 diabetes mellitus, abnormal blood lipids, sleep apnea, and reduced physical fitness, but also psychological problems such as reduced levels of quality of life and self-esteem, negative body image, depression and mental distress $[14,15]$.

Changes in health related behavior such as increased consumption of foods and drinks high in sugar and/or fat, and a more sedentary lifestyle are generally discussed to be underlying factors of rising prevalence rates of overweight and obesity $[16,17]$. Both healthy and unhealthy behaviors in childhood have been known to track into later life [18-20] and may increase or decrease the likelihood for excessive weight gain.

To date, many epidemiologic studies examining associations between obesity and dietary and sedentary/physical activity behaviors have focused on assessing Body Mass Index (BMI) at one point in time, ignoring the dynamic changes of BMI over time and the diversity in patterns that may emerge during the natural development of children. Recent developments in statistical techniques make it possible to study the potential heterogeneity in the development of BMI during childhood [21-23]. The assumption is made that, instead of the existence of a single developmental curve in the study population, individuals belong to distinct subpopulations with different developmental trajectories, and that individual children may have different pathways in the development of overweight.

When investigating population heterogeneity a personcentered approach can offer more insight than a variablecentered approach. Person-centered techniques aim to group similar individuals based on their characteristics, focusing on relationships among individuals instead of on relationships among variables such as in regression models and structural equation models [21]. Latent class models, and specifically latent class growth (mixture) models (LCGMM) are examples of such a technique [21-26].

Identifying groups of individuals following similar patterns of BMI over age in distinct BMI trajectories may be useful because it may help to identify different pathways of overweight onset and development during childhood and increase our understanding of the mechanisms underlying increasing trends in overweight prevalence. This could give us insight in developing specific interventions for specific subgroups based on these distinct trajectories, given that the effects of many interventions on overweight prevention have been limited, especially on the long term [27].

There have been a number of studies that have prospectively explored BMI trajectories in the period of (early) childhood [28-33], and only one of these examined associations between BMI trajectories and health behavior (sleep duration) in childhood [34]. This exploratory study will give us insight in the possible existence of BMI trajectories in our study population and whether certain health behaviors in early childhood are associated with unfavorable changes in BMI. In the literature we often find a normative/stable trajectory, comprising the larger part of the sample, a progressively overweight trajectory and a progressively overweight but stabilizing trajectory [35]. In addition, the existence of a stable high BMI trajectory is reported in many studies of BMI trajectories [36-39]. We therefore hypothesize the possible existence of multiple overweight trajectories, including a stable high trajectory.

We will address these issues applying LCGMM techniques using data from the ChecKid study, covering 
childhood from 4 years to 12 years of age. We aim to 1 ) identify distinct BMI trajectories with an exploratory approach and 2) examine associations between dietary, sedentary and physical activity behaviors and these distinct trajectories.

\section{Methods}

\section{Study population and design}

The ChecKid study is a dynamic cohort study of primary school children in the ages of 4 to 12 years in the city of Zwolle, the Netherlands. The objectives of ChecKid are to investigate trends in overweight and to examine health behaviors related to childhood overweight and obesity and determinants of these behaviors within families, schools and neighborhoods. ChecKid is part of an integrated approach in which quantitative and qualitative monitoring research and environmental scans support the development, implementation and evaluation of tailored community wide interventions.

This study commenced in 2006 and in 2006, 2009 and 2012 every primary school in the city of Zwolle $(n=51 / 43$ / 43) was invited to participate in the study. When a school agreed to be included in the study, all children attending the school (4-12 years) and their parents were invited to participate by means of letters distributed via the schools. Respectively $80 \%, 79 \%$ and $81 \%$ of the schools were willing to participate in 2006, 2009 and 2012. When schools did not want to participate, it was mostly because of other priorities. Participating schools were equally spread over all neighborhoods in Zwolle. Measurements took place every three years, during 3 weeks, in October and November of 2006, 2009 and 2012, thus indicating a follow up time of six years.

Eligible children included those who had an anthropometric measurement (height and weight) and whose parents filled in a self-report questionnaire about the reported behaviors in 2006, in 2009 and in 2012. Total response in 2006 was 4,072 children (49\%) of whom anthropometric measurements and questionnaires were available, in 2009 this was 3,026 (35\%) and in 2012 5,849 (61\%). The dynamic design of the study entailed that every three years the total population at every school was invited to participate in the study. Due to this design only children who were in class 1,2 and 3 in 2006 ( $n=1,599$; age range 4.0 to 7.0 years) can be included as a cohort. No new participants were recruited in 2009 and 2012. Exclusion criteria for participants were not being sufficient in the Dutch language, being older than 13 years of age at follow up, attending special education, and not living in the city of Zwolle.

For the estimation of the trajectories at least two measurements were included. Because we felt that it was necessary to have a "true" measurement at baseline instead of an estimation of the trajectory, we used data of all children with a baseline measurement in 2006. Besides baseline, a minimum of 1 measurement in 2009 and/or in 2012 was included. Because of high response rates in 2006 and 2012, none of the participants had a missing measurement during these years, meaning we only have missing measurements in 2009 in our dataset. Two hundred forty-three (39.6 \%) of the participants had only 2 measurements. This leaves us with a sample of 613 children, who were included and completed follow-up. Mean age at baseline is 5.1 years (range 4.0 to 7.0 years), at the second wave it is 8.1 years (range 7.0 to 9.8 years) and in 201211.1 years (range 10.0 to 12.9 years).

All parents gave written informed consent and medical ethical approval was obtained from the Medical Ethics Committee of the VU University Medical Centre.

\section{Measures}

\section{BMI}

Height, weight and waist circumference were measured according to protocol [40] by trained students. BMI was calculated as weight in kilograms divided by height in meters squared. The children's BMI cut-off points defined by Cole et al. were used to define thinness, healthy weight, overweight and obesity $[41,42]$. Because an increase in BMI during childhood is a feature of natural growth, we calculated individual age and gender specific BMI standard deviation scores (SDS) using the data from the National Growth Study 1997 as the reference population [7].

\section{Demographic variables and health behaviors}

We obtained information on the child's health behavior (dietary, physical activity and sedentary behavior) during weekdays and weekends separately by self-administered questionnaires completed by one of the parents. We used information on the child's health behavior during weekdays, as we were especially interested in finding indications for interventions that could possibly be implemented or supported in a school setting. Participation in organized sports is the only variable which was assessed during weekdays and weekends combined ( $>1 \mathrm{~h}$ a week) because a lot of sports activities are pursued during the weekend. The questionnaire for parents included sociodemographic variables such as the child's age, gender, postal code, ethnicity (assessed by country of birth of both parents) and socio-economic status (SES) (assessed by educational level parents). Parents' self-reported body weight and height data were used to calculate their BMI. Existing validated questionnaires on health behavior were used for the design of this questionnaire [43, 44]. As the questionnaires were originally designed for secondary school students, the questionnaires were adjusted for primary school children in this study [44]. Details of the ChecKid questionnaires are described elsewhere [45]. 
Dietary intake was measured by exploring frequency and amount of fruit intake, vegetable intake and frequency and amount of sugared drink consumption. Consumption of vegetables was dichotomized as eating vegetables daily (5 days a schoolweek) vs not daily ( $<5$ days a schoolweek). Fruit consumption was dichotomized as less than 2 portions a weekday ( $<2$ portions) vs 2 or more portions a weekday ( $\geq 2$ portions). This was done according to guidelines issued by the Dutch Nutrition Centre [46, 47]. The consumption of sugared drinks (fruit juices, soft drinks and sweetened milk drinks) was dichotomized as 3 or less glasses of sugared drinks per weekday ( $\leq 3$ glasses) versus more than 3 glasses of sugared drinks per weekday ( $>3$ glasses), according to guidelines used in previous studies $[45,48]$.

Sedentary behavior was measured by investigating total screen time (TV viewing and using the computer) and TV viewing separately. Parents were asked to report frequency and duration of time (in categories) spent watching TV or using the computer. Averages were calculated by multiplying the number of days that the child spent on the behavior by the mid-category values of duration of the item in 5 categories: <0.5, 0.5-1, 1-2, 2-3, and $>3 \mathrm{~h}$ a day, and dividing this by the number days per week. Screentime and TV viewing were both dichotomized as less than $2 \mathrm{~h}(<2 \mathrm{~h})$ or $2 \mathrm{~h}$ or more $(\geq 2 \mathrm{~h})$ per weekday.

Physical activity was measured by investigating time spent on outside play and in organized sports. Parents were asked to report frequency and duration of time (in categories) spent on outside play or participation in organized sports. Averages were calculated by multiplying the number of days that the child spent on the behavior by the mid-category values of duration of the item in 5 categories: $<0.5,0.5-1,1-2,2-3$, and $>3 \mathrm{~h}$ a day, and dividing this by the number of days per week. Outside play was dichotomized according to the Dutch standard for exercise for children as playing outside $1 \mathrm{~h}$ or more $(\geq 1 \mathrm{~h})$ and less than $1 \mathrm{~h}(<1 \mathrm{~h})$ per weekday [49]. Participation in organized sports was dichotomized as less than $1 \mathrm{~h}(<1 \mathrm{~h})$ and $1 \mathrm{~h}$ or more $(\geq 1 \mathrm{~h})$ per week.

\section{Statistical analyses}

Statistical analyses were conducted using the Mplus 7.11 and the PASW 20.0 software packages. Descriptive statistics were used to investigate population characteristics and differences in behaviors over time, using Chi square and t-tests.

\section{Estimation of BMI trajectories}

Longitudinal BMI trajectories were analyzed with latent class growth mixture modeling [21, 25, 50, 51], LCGMM. LCGMM is a contemporary longitudinal technique in obesity research. The underlying aim of the technique is to capture the heterogeneity in BMI development over time in $k$ number of subgroups (or classes), each with a distinct BMI trajectory shape. Each class has its own growth parameters (i.e., intercept and linear slope), where the intercept represents the average BMI-value at baseline and the slope is indicative of the rate at which the BMIvalues change over time.

Various LCGMM models were run before we chose a final model. First, several linear LCGMM with fixed intercept and slope variance within classes were investigated. Next, quadratic slopes were added to the model allowing for curved developmental patterns. The timescores were based on the follow-up measurements and were held equal across time $(0,1,2)$. To determine the optimal number of classes, a common forward procedure $[26,35]$ was performed, starting with a one class solution (i.e., assuming all individuals follow a similar BMI trajectory over time), then adding more classes one at a time to investigate whether or not the model fit improves due to the additional class(es). To explore the possibility of different trajectories by gender, these steps were initially performed separately in boys and girls. Because the trajectories were very similar in nature in both girls and boys, as also found in Pryor et al. [31] and Magee [34], the analyses were performed on the total sample. The results of these analyses will be reported in this paper.

To compare fit between subsequent models, we used several model fit indices and other criteria, as suggested by the literature [52]. First, we used two model fit indices; the Bayesian Information Criterion (BIC) and the Bootstrapped Likelihood Ratio Test (BLRT). The BIC $[53,54]$ is common in latent class models and considers both the likelihood of the model as well as the number of parameters in the model. A lower value is indicative of a better fitting model. The BLRT [55] provides a p-value, comparing a model with $k-1$ classes with a model with $k$ classes. The BLRT has been shown to be a very consistent indicator of the optimal number of classes [56]. To further look at the optimal number of classes, we also took the posterior probabilities into account. Children were assigned to their most-likely class based on these probabilities. The probability for the class to which a child is assigned to should be considerably higher that the probabilities for the other classes to make the classes easily distinguishable from each other.

Finally, we considered the usefulness of the models in practice by examining the shape of the trajectories and the sample size of each class. We ran several quadratic models, forcing all within-class variances at zero (Nagin type). Although the BIC for the 2-class model was slightly lower (3579.191 versus 3607.081 for the linear model), the class size and the trajectory shapes were similar. The linear GMM included estimated (free) intercept variances within classes (Mplus default) and the slope variance was 
forced at zero (Nagin type model). We based these choices on the comparison of model fit indices described earlier. We rejected models with clinically uninterpretable classes as well as models including classes with a sample size $<1 \%$ of the total sample [26].

\section{Associations between health related behaviors and BMI trajectories}

The first step of the analyses provides us with a classifitheir BMI trajectory. This classification is coded as a categorical variable with $k$ number of categories, or classes. To study the possible associations between the classes and behaviors at baseline and the possible associations between the classes and behaviors at last measurement, several logistic regression models were conducted, in which the dichotomized behavior variable is the outcome measurement and class membership the independent variable. First crude analyses were performed. Second, adjusted analyses were carried out, controlling for potential confounding effects of gender, SES and ethnicity. Separate logistic regression models were performed for associations with behaviors at baseline in 2006 and for associations with behaviors in 2012 .

Although our primary aim was to examine associations between behaviors at baseline and the trajectories to explore the predictive value of the behaviors, we also examined associations between behaviors at last measurement in 2012 and the trajectories. We wanted to explore the cross-sectional association between current behavior and BMI trajectory as parallel outcome. As behavior is known to track throughout life [18-20] it is modifiable, and as children become more autonomous in their behavior with age, we therefore think it is valuable to explore associations between health behavior at last measurement and the trajectories.

\section{Missing data}

Missing value percentages in the behavior variables were $13.9 \%$ (TV viewing), $14.2 \%$ (vegetable consumption), $14.5 \%$ (outside play), $15.2 \%$ (participation in organized sports), $15.5 \%$ (screentime), $20.6 \%$ (fruit consumption) and $27.7 \%$ (consumption of sugared drinks) in 2006, and $0.8 \%$ (consumption of vegetables), $1.1 \%$ (consumption of sugared drinks), $3.3 \%$ (fruit consumption), $4.4 \%$ (TV viewing), $5.4 \%$ (outside play), $5.4 \%$ (participation in organized sports), and $12.7 \%$ (screentime) in 2012. Because overweight children and children with a low SES background more often had a missing value on these variables, missing data were not completely at random. The authors used the multiple imputation procedure in PASW 20.0 software to impute the missing data. The variables: BMI of both parents, ethnicity, gender and age were included in the imputation models, and data were imputed ten times using the 'Predictive Mean Matching '(PMM) procedure. For comparison reasons, data were also imputed five, twenty and fifty times using the same method, resulting in a total of 40 imputed datasets. All analyses were performed on the original data and on the imputed datasets. No major differences were found in the direction of the found associations, nor in the slopes or p-values.

\section{Results}

Number of BMI trajectories: model selection

Based on the BIC the "best fitting" model (the model with the lowest value) was the two class linear model, although the BIC of the three class linear model was almost the same (a difference of 1 point) (Table 1). Improvement of at least 10 points indicates a significant improvement of the model, $[53,54]$ indicating that based on the BIC, the two and three class linear models had equivalent fit. Based on the posterior probabilities however, the results of the three-class model were not robust enough, indicating lack of discriminatory power. The fit information of the BIC, BLRT, posterior probabilities and number of subjects per class are presented in Table 1.

\section{Sensitivity analysis}

Because the two class and three class model both had equivalent fit, and because it was difficult to determine a clear normative, stable trajectory we decided to explore the three class model in an extra sensitivity analysis. We found a stable high BMI SDS trajectory $(n=212)$, a decreasing BMI SDS trajectory $(n=346)$, and a steeply increasing BMI SDS trajectory $(n=55)$. The three-class model did not yield a clear normative trajectory either, and when we compare the steeply increasing and increasing trajectories in the 3 class model using descriptive analyses of characteristics of the study population and the health behaviors, we find that they are nearly the same, see Additional file 1: Table S1 and Table S2 and Additional file 2: Figure S1. This indicates lower posterior probabilities and unclear class distinctiveness.

After taking into account the substantive interpretation of the two models and objectives of the analyses, a final decision for the model with two classes was made, showing a decreasing BMI SDS trajectory $(n=416)$ and an increasing BMI SDS trajectory $(n=197)$. Figure 1 shows mean trajectories of the final model. In our data we could find no children who maintained BMI SDS scores of 0 in 2006 and in 2012, thus supporting our finding lacking a normative group.

\section{Demographics and health related behaviors in the BMI trajectories}

The prevalence of overweight (including obesity) in the total study population was $7.2 \%$ in 2006 , and $9.8 \%$ in 
Table 1 Model fit indices of LCGMM

\begin{tabular}{lllll}
\hline Number of classes & Bayesian Information Criterion & $\begin{array}{l}\text { Bootstrapped likelihood } \\
\text { ratio test (BLRT) }\end{array}$ & $\begin{array}{l}\text { Average posterior } \\
\text { probability (min-max) }\end{array}$ & $\begin{array}{l}\text { Number of subjects } \\
\text { per class }\end{array}$ \\
\hline 1 & 3631.500 & - & 1.00 & 613 \\
2 & 3607.081 & $P<0.001$ & $0.827(0.784-0.869)$ & $416 / 197$ \\
3 & 3608.926 & $P<0.001$ & $0.739(0.678-0.825)$ & $212 / 346 / 55$ \\
4 & 3618.315 & $P=0.2857$ & $0.823(0.709-0.918)$ & $204 / 23 / 4 / 382$ \\
5 & 3626.946 & $P=0.05$ & $0.771(0.709-0.826)$ & $353 / 23 / 25 / 200 / 12$ \\
\hline
\end{tabular}

2012 (Table 2). Of the children in the increasing BMI SDS trajectory, the prevalence of overweight (including obesity) increased from $8.7 \%$ in 2006 to $22.3 \%$ in 2012, in contrast to the decreasing BMI SDS trajectory where the prevalence decreased from $6.5 \%$ in 2006 to $1.7 \%$ in 2012.

The increasing BMI SDS trajectory had a statistically significantly higher percentage of participants of nonwestern ethnicity and lower SES than the decreasing BMI SDS trajectory. In the increasing BMI SDS trajectory maternal overweight prevalence was significantly higher than in the decreasing trajectory, both at baseline in 2006 and at follow-up. Paternal overweight prevalences did not differ significantly at baseline, but at follow-up the prevalence of overweight fathers was significantly higher in the increasing BMI SDS trajectory.

Children in the increasing BMI SDS trajectory were more likely to play outside than children in the decreasing BMI SDS trajectory in both 2006 and 2012 (Table 3), but participated less often in organized sports; a statistically significant difference in the participation of organized sports was apparent in 2012. Sedentary behaviors, such as TV viewing and screentime more than $2 \mathrm{~h}$ per day were both more common in the increasing BMI SDS trajectory.

\section{Associations between BMI trajectories and health related behaviors at baseline}

Logistic regression analyses were performed and adjustments for potential confounders were carried out based on prior knowledge in published literature. After adjustment for gender, SES and ethnicity, the children in the increasing BMI SDS trajectory scored higher odds for not eating the recommended vegetable intake (OR, 1.1; $95 \%$ confidence interval (CI) 0.8 to 1.7 ), drinking more than the recommended amount of sugared drinks (OR, 1.2; $95 \%$ CI 0.8 to 1.8), participating in organized sports less than recommended (OR, 1.1; $95 \%$ CI 0.7 to 1.6), and TV viewing more than $2 \mathrm{~h}$ daily (OR, 1.2; $95 \% \mathrm{CI}$ 0.4 to 3.6) than those in the decreasing BMI SDS trajectory, though these results were not significant (Table 4). The OR for maternal overweight status was significantly higher for children in the increasing BMI SDS trajectory compared to the children in the decreasing BMI SDS trajectory (OR, 2.9; $95 \%$ CI 1.9 to 4.3 ).

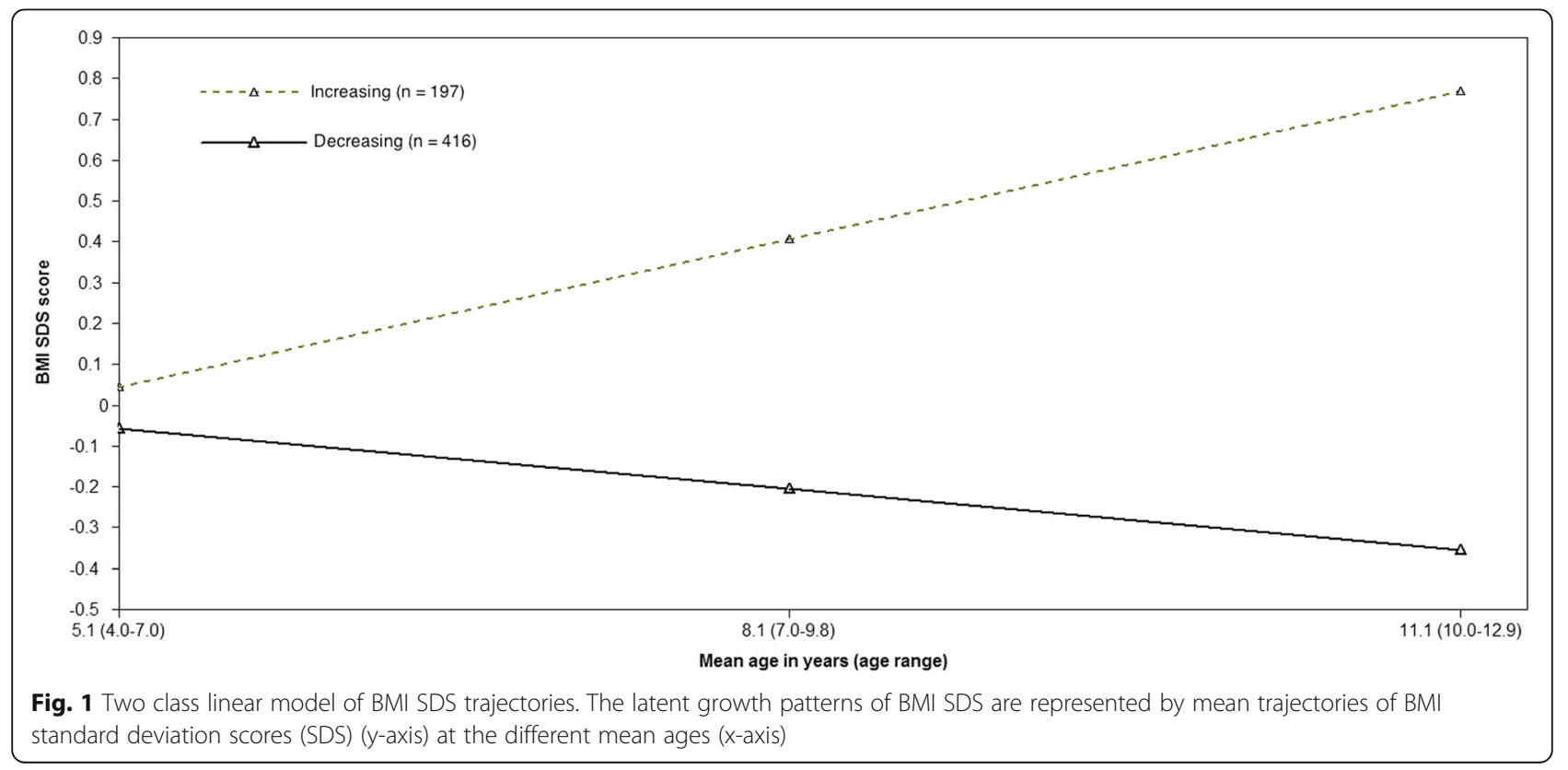


Table 2 Characteristics of the study population

\begin{tabular}{|c|c|c|c|c|}
\hline \multirow{2}{*}{$\begin{array}{l}\text { Variables of interest [\%] } \\
\text { or [mean (SD)] }\end{array}$} & \multicolumn{3}{|l|}{ BMI trajectories } & \multirow[t]{2}{*}{$P$-value ${ }^{a}$} \\
\hline & Increasing $(N=197)$ & Decreasing $(N=416)$ & Total sample $(N=613)$ & \\
\hline Gender \% male & 54.8 & 48.1 & 50.2 & \\
\hline \multicolumn{5}{|l|}{ SES } \\
\hline low & 18.2 & 9.3 & 12.1 & $* *$ \\
\hline middle & 26.6 & 20.3 & 22.3 & $* *$ \\
\hline high & 55.2 & 70.4 & 65.6 & $* *$ \\
\hline Ethnicity - \% non-western & 14.4 & 5.8 & 8.5 & $* *$ \\
\hline \multicolumn{5}{|l|}{ BMl } \\
\hline 2006 & $15.8(1.5)$ & $15.5(1.2)$ & $5.6(1.3)$ & \\
\hline 2009 & $17.1(2.4)$ & $15.4(1.6)$ & $16.0(2.0)$ & $* *$ \\
\hline 2012 & $19.8(2.6)$ & $16.6(1.6)$ & $17.6(2.5)$ & $* *$ \\
\hline \multicolumn{5}{|l|}{ Waist circumference } \\
\hline 2006 & $53.6(4.2)$ & $53.3(5.8)$ & $53.4(5.4)$ & \\
\hline 2009 & $61.6(7.0)$ & $57.3(4.0)$ & $58.7(5.5)$ & $* *$ \\
\hline 2012 & $71.8(8.1)$ & $62.8(5.1)$ & $65.7(7.5)$ & $* *$ \\
\hline \multicolumn{5}{|l|}{ Weight status child (\%) } \\
\hline \multicolumn{5}{|l|}{ Thinness } \\
\hline 2006 & 9.6 & 10.8 & 10.4 & * \\
\hline 2009 & 5.0 & 17.9 & 13.8 & $* *$ \\
\hline 2012 & 0.0 & 15.6 & 10.6 & $* *$ \\
\hline \multicolumn{5}{|l|}{ Healthy weight } \\
\hline 2006 & 81.1 & 82.7 & 82.4 & * \\
\hline 2009 & 77.3 & 77.3 & 77.3 & $* *$ \\
\hline 2012 & 73.1 & 82.1 & 79.6 & $* *$ \\
\hline \multicolumn{5}{|c|}{ Overweight (including obesity) } \\
\hline 2006 & 8.7 & 6.5 & 7.2 & * \\
\hline 2009 & 17.6 & 4.8 & 8.9 & $* *$ \\
\hline 2012 & 26.9 & 1.7 & 9.8 & $* *$ \\
\hline \multicolumn{5}{|l|}{ Weight status parent } \\
\hline \multicolumn{5}{|l|}{ BMI mother (\% overweight) } \\
\hline 2006 & 46.0 & 20.7 & 29.6 & $* *$ \\
\hline 2012 & 40.7 & 25.3 & 30.2 & ${ }^{* *}$ \\
\hline \multicolumn{5}{|l|}{ BMI father (\% overweight) } \\
\hline 2006 & 51.4 & 46.6 & 48.1 & \\
\hline 2012 & 58.2 & 45.3 & 49.4 & * \\
\hline
\end{tabular}

Abbreviations: BMI body mass index (calculated as weight in kilograms divided by height in metres squared), SDS standard deviation scores, SES socioeconomic status, $S D$ standard deviation

${ }^{a} P$-values determined using $X^{2}$ test (categorical variables or $T$-test (continuous variables): ${ }^{*} p<0.05$ for difference between trajectories; ${ }^{* *} p<0.001$ for difference between trajectories

\section{Associations between BMI trajectories and health related behaviors in 2012}

After adjustment, the children in the increasing BMI SDS trajectory scored higher odds in 2012 for drinking more than the recommended amount of sugared drinks (OR, 1.2; 95 \% CI 0.8 to 1.8 ), participating in organized sports less than recommended (OR, 1.3; $95 \%$ CI 0.8 to 2.0 ), and more than recommended use of screentime (OR, 1.3; $95 \%$ CI 0.9 to 1.8 ) than those in the decreasing BMI SDS trajectory, though these results were also not significant. The OR for maternal (OR, 1.8; 95 \% CI 1.2 to 2.6) as well as for paternal overweight status (OR, 1.6; 
Table 3 Health related behaviors in the BMI SDS trajectories

\begin{tabular}{|c|c|c|c|c|}
\hline \multirow{2}{*}{$\begin{array}{l}\text { Variables of } \\
\text { interest [\%] }\end{array}$} & \multicolumn{3}{|l|}{ BMI trajectories } & \multirow[t]{2}{*}{$P$-value } \\
\hline & Increasing $(N=197)$ & Decreasing $(N=416)$ & Total sample $(N=613)$ & \\
\hline \multicolumn{5}{|c|}{ Dietary behaviors } \\
\hline \multicolumn{5}{|c|}{ Vegetable intake $<5$ days a week } \\
\hline 2006 & 60.8 & 55.5 & 57.1 & \\
\hline 2012 & 52.8 & 59.8 & 57.4 & \\
\hline \multicolumn{5}{|c|}{ Fruit $<2$ portions a day } \\
\hline 2006 & 72.5 & $72.9 * * * b$ & $72.8^{* * *}$ & \\
\hline 2012 & 76.0 & $82.2 * * *$ & $80.4^{* * *}$ & \\
\hline \multicolumn{5}{|c|}{ Sugared drinks $>3$ glasses a day } \\
\hline 2006 & $54.0 * * *$ & $46.0 * * *$ & $48.6^{* * *}$ & \\
\hline 2012 & $40.2 * * *$ & $33.8 * * *$ & $35.9^{* * *}$ & \\
\hline \multicolumn{5}{|c|}{ Physical activity behaviors } \\
\hline \multicolumn{5}{|c|}{ Outside play $<1 \mathrm{~h}$ a day } \\
\hline 2006 & $53.6(4.2)$ & $53.3(5.8)$ & $53.4(5.4)$ & \\
\hline 2012 & $71.8(8.1)$ & $62.8(5.1)$ & $65.7(7.5)$ & \\
\hline \multicolumn{5}{|c|}{ Organized sports $<1 \mathrm{~h}$ a week } \\
\hline 2006 & $69.1 * * *$ & $66.5^{* * *}$ & $67.3^{* * *}$ & \\
\hline 2012 & $20.9 * * *$ & $14.7^{* * *}$ & $16.7^{* * *}$ & * \\
\hline \multicolumn{5}{|c|}{ Sedentary behaviors } \\
\hline \multicolumn{5}{|c|}{ TV viewing $>2 \mathrm{~h}$ a day } \\
\hline 2006 & $4.1^{* * *}$ & $2.8^{* * *}$ & $3.2 * * *$ & \\
\hline 2012 & $14.9 * * *$ & $9.9 * * *$ & $11.5^{* * *}$ & \\
\hline \multicolumn{5}{|c|}{ Screentime $>2 \mathrm{~h}$ a day } \\
\hline 2006 & $6.2 * * *$ & $5.6 * * *$ & $5.8 * * *$ & \\
\hline 2012 & $65.0 * * *$ & $57.2 * * *$ & $59.7^{* * *}$ & \\
\hline
\end{tabular}

Abbreviations: $B M I$ body mass index (calculated as weight in kilograms divided by height in metres squared), SDS standard deviation scores

${ }^{\text {a }} P$-values determined using $X^{2}$ test : ${ }^{*} p<0.05$ for difference between trajectories

${ }^{b} P$-values determined using $X^{2}$ test: ${ }^{* *} p<0.001$ for differences in health related behaviors between baseline and follow-up

$95 \%$ CI 1.1 to 2.4) was significantly higher for children in the increasing BMI SDS trajectory compared to the children in the decreasing BMI SDS trajectory.

\section{Changes in health related behaviors between 2006 and 2012 in the BMI trajectories}

Chi square tests were performed to explore differences in behaviors between 2006 and 2012 (Table 3), and for both trajectories a statistically significant increase was found in participation in organized sports, and in TV viewing/ screentime more than $2 \mathrm{~h}$ daily, as well as a statistically significant decrease in the consumption of sugared drinks.

In the decreasing BMI SDS trajectory fruit consumption decreased significantly, and outside play increased significantly (see Fig. 2).

\section{Discussion}

Over the past years the existence of heterogeneity within a study sample has been acknowledged, signaling the need for more research regarding developmental pathways in diverse study populations in diverse fields of research. Most studies regarding the development of overweight in childhood have investigated the development of overweight using BMI at one point in time, and categorize the study population into overweight and non-overweight children on the basis of their BMI at that point. This a priori categorization of the study participants into predefined groups on basis of their overweight status, is contrary to the categorization with LCGMM, which is on basis of developmental trajectory of the participants.

Use of latent class models have led to discussion in current literature in epidemiology. There are two different methods available: latent class mixture modelling (LCMM), which assumes no within-class variation (variance in each class is fixed to zero) and LCGMM models which allows estimation of within-class variance. LCGMM allows for more heterogeneity in the model because of this estimation 
Table 4 Logistic regressions showing associations between class membership and behaviors at baseline (2006) and at follow-up (2012)

\begin{tabular}{|c|c|c|c|c|}
\hline & \multicolumn{2}{|c|}{ Crude analyses, unadjusted } & \multicolumn{2}{|c|}{ Adjusted for gender, SES and ethnicity } \\
\hline & $\begin{array}{l}2006 \\
\text { OR (95 \%-Cl) }\end{array}$ & $\begin{array}{l}2012 \\
\text { OR (95 \%-Cl) }\end{array}$ & $\begin{array}{l}2006 \\
\text { OR (95 \%-Cl) }\end{array}$ & $\begin{array}{l}2012 \\
\text { OR (95\%-Cl) }\end{array}$ \\
\hline \multicolumn{5}{|l|}{ Dietary behaviors } \\
\hline Vegetable intake $<5$ days a week & $1.2(0.8-1.8)$ & $0.8(0.5-1.1)$ & $1.1(0.8-1.7)$ & $0.7(0.5-1.0)^{*}$ \\
\hline Fruit $<2$ portions a day & $1.0(0.7-1.5)$ & $0.7(0.4-1.0)$ & $1.0(0.7-1.6)$ & $0.8(0.5-1.2)$ \\
\hline Sugared drinks $>3$ glasses a day & $1.4(1.0-2.0)$ & $1.3(0.9-1.9)$ & $1.2(0.8-1.8)$ & $1.2(0.8-1.8)$ \\
\hline \multicolumn{5}{|l|}{ Physical activity behaviors } \\
\hline Outside play $<1 \mathrm{~h}$ a day & $0.9(0.6-1.3)$ & $0.8(0.6-1.2)$ & $0.8(0.6-1.2)$ & $1.0(0.7-1.4)$ \\
\hline Organized sports $<1 \mathrm{~h}$ a week & $1.13(0.76-1.68)$ & $1.5(1.0-2.4)$ & $1.1(0.7-1.6)$ & $1.3(0.8-2.0)$ \\
\hline \multicolumn{5}{|l|}{ Sedentary behaviors } \\
\hline TV viewing $>2$ h a day & $1.5(0.5-4.8)$ & $1.6(1.0-2.7)$ & $1.2(0.4-3.6)$ & $1.3(0.8-2.3)$ \\
\hline Screentime $>2 \mathrm{~h}$ a day & $1.1(0.5-2.6)$ & $1.4(1.0-2.0)$ & $1.0(0.4-2.3)$ & $1.3(0.9-1.8)$ \\
\hline \multicolumn{5}{|l|}{ Weight status parent } \\
\hline BMI mother - overweight & $3.0(2.0-4.5)^{* *}$ & $2.0(1.4-3.0)^{* *}$ & $2.9(1.9-4.3)^{* *}$ & $1.8(1.2-2.7)^{* *}$ \\
\hline BMI father - overweight & $1.2(0.8-1.8)$ & $1.7(1.2-2.4)^{*}$ & $1.2(0.8-1.7)$ & $1.6(1.1-2.4)^{*}$ \\
\hline
\end{tabular}

Abbreviations: BMI body mass index (calculated as weight in kilograms divided by height in metres squared), SES socioeconomic status, OR odds ratios, $C I$ confidence interval

Decreasing BMI SDS group was set as reference

$P$-values: ${ }^{*} p<0.05$ for difference between trajectories; ${ }^{* *} p<0.001$ for difference between trajectories

of within-class variance, and because of this, in many situations models do not converge [57].

There is also discussion on how to decide on the final number of classes based on the information provided by model fit indices [52], the main point of discussion being the inconsistency between these model fit indices [26, 52]. This process is guided by statistical fit indices as well as usefulness of the model for practice, and could possibly lead to over or underestimation of the true number of trajectories or groups in the study population [52].
In this longitudinal study, we identified and described two BMI SDS trajectories in 613 children from 4 to 12 years of age. The identified patterns of BMI SDS showed that at 4 years of age for the two groups of children with different trajectories there were no notable differences in mean BMI SDS but at follow-up six years later two diverging patterns had emerged. The largest group (68\%) was characterized by stable decreasing mean BMI SDS scores and in the other group (32\%) the mean BMI SDS scores showed an increase. The increasing BMI SDS trajectory consisted statistically significantly of

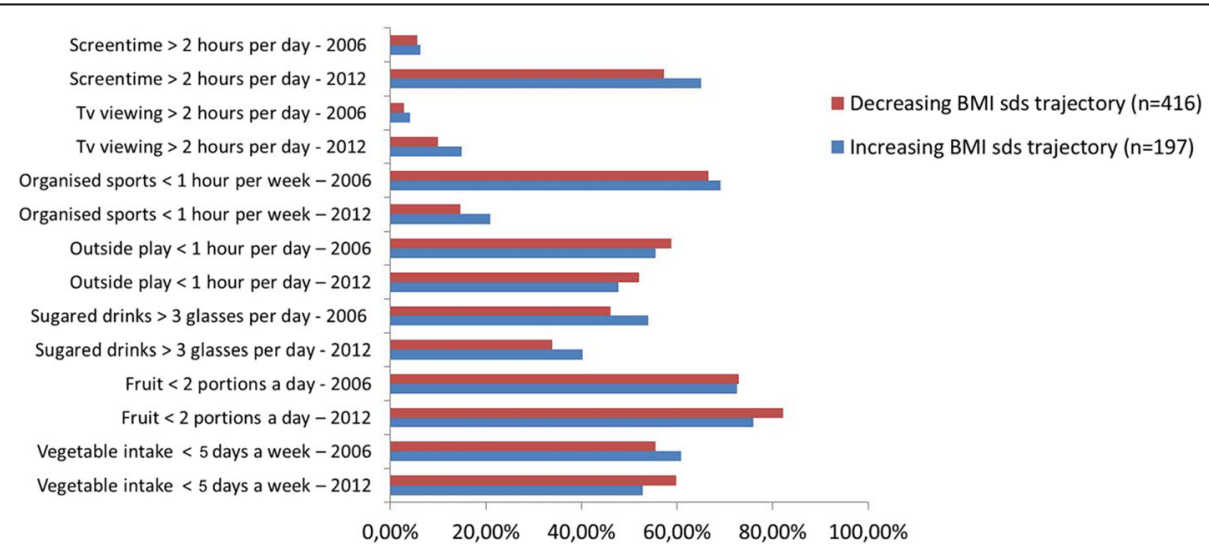

Fig. 2 Changes in health related behaviors between 2006 and 2012 in the two BMI SDS trajectories. Depicted are the percentage of children who display the examined unhealthy behaviors in 2006 in each BMI SDS trajectory and the percentage of children who display these unhealthy behaviors in 2012, visualizing the change in behaviors per trajectory in six years time 
more participants of lower and middle SES and more of non-western ethnicity.

Maternal overweight status increased the risk for being in the increasing BMI SDS trajectory, as did paternal overweight status at follow-up. In our study the odds of having an overweight mother were 2.87 times higher at baseline and 1.81 times higher at follow-up for children in the increasing BMI SDS trajectory compared to children in the decreasing trajectory. Unhealthy behaviors at both baseline and follow-up were associated with BMI trajectories. For instance, being in the increasing BMI SDS trajectory was associated with the following behaviors; drinking sugared drinks $>3$ glasses per day, participation in organized sports $<1 \mathrm{~h}$ per week, and TV viewing $>2 \mathrm{~h}$ per day, though participation in organized sports at follow-up was the only significant result. Being in the decreasing BMI SDS trajectory was associated with outside play $<1 \mathrm{~h}$ per day at both baseline and follow-up, though also not significant.

When exploring differences in health behaviors between 2006 and 2012, a statistically significant increase for all groups is found in participation in organized sports, and in TV viewing/screentime more than $2 \mathrm{~h}$ daily, as well as a statistically significant decrease in the consumption of sugared drinks. Because older children are more autonomous than younger children these findings may reflect an effect of time or age.

In another study that investigates BMI trajectories in childhood and associations with health behavior (sleep duration), longitudinal associations were found between sleep duration and BMI in children with early onset obesity [34]. Other studies concerning associations between unhealthy behavior and childhood overweight confirm our findings concerning TV-viewing and screentime $[45,44,48]$, sugared drinks $[45,48]$ and participation in organized sports [44, 48], though these were not longitudinal by nature.

\section{BMI trajectories in childhood}

There have been a number of studies that have prospectively explored BMI trajectories in the period of (early) childhood $[28-34,56]$. We found three studies on BMI trajectories and associations with early life risk factors. One study shows three trajectories of BMI (low-stable, moderate, and high-rising) in children between 5 months and 8 years of age [31]. The low-stable and moderate trajectory groups showed a similar shaped stable developmental pattern, whereas the high rising group was characterized by an increasing average BMI, which exceeded international cut-off values for obesity by age 8 years. Maternal smoking and maternal BMI were important risk factors for the high rising group. These risk factors were also found in another study which also identified three overweight trajectories: early onset overweight, late onset overweight, and never overweight in children from 2 years to 12 years old [33]. The early onset group showed early onset of overweight which persisted throughout childhood, and the late onset group had a moderately high probability of overweight at 2 years, low probability at age 4 and 6 years, but growing probability of overweight after age 8 years. The never overweight group had a low probability of developing overweight throughout childhood. In another study which identified four BMI trajectories in children from 4 to 11 years old (high-risk overweight, early onset overweight, later onset overweight and healthy weight), maternal overweight, maternal smoking and high birth weight were associated with the high-risk overweight trajectory [29]. In a follow-up study the same authors found three trajectories (healthy weight, early onset obesity, and later onset obesity) and found longitudinal associations between the early onset obesity trajectory and sleep duration [34].

In most studies concerning BMI trajectories (in childhood), besides a progressively overweight trajectory and a progressively overweight but stabilizing trajectory, a normative/stable trajectory can be found [30-32, 35]. In our study the two-class model, which had the best fit, did not comprise such a normative trajectory. Instead, most of the sample (68\%) was identified as a decreasing BMI SDS trajectory. We also explored the three-class model in an extra sensitivity analysis, but in this model no normative or stable trajectory was found either.

In a study which examined socioeconomic equalities in childhood trajectories of BMI and overweight a longitudinal tendency was found, showing two longitudinal influences: decreasing BMI in the more advantaged populations (high SES), coupled with simultaneously worsening rates of overweight in the more disadvantaged (low SES) [58]. By age 10-11 socioeconomic differences in mean BMI z-scores already present at age 4-5 had more than doubled, reflecting decreasing mean BMI among advantaged rather than increasing means among disadvantaged children. In our study the decreasing trajectory consists of significantly more children of high SES, supporting this previous finding, thus highlighting the importance of SES in the development of overweight in childhood.

\section{Strengths and limitations of the study}

Our study is strengthened by the high participation rates and the equal spread of participating schools in one city, as well as all children being measured during a three week period according to protocol. The prospective repeated measurements add to the strengths of this study. Some limitations of our study can be identified. A potential limitation is that only three measurements of BMI were used to obtain BMI developmental trajectories. 
The need to impute relative high percentage missing values of low SES and overweight children on the behavior variables at baseline could indicate possible bias, although our analyses on the imputed on non-imputed datasets did not yield different results. The use of parental self-report questionnaires on health behaviors can possibly lead to social desirability and difficulties recalling the actual behaviour of the child, especially if this behavior takes place outside the home [59]. This could lead to over or underestimation of the child's behavior, possibly influencing the generalization of the results. Recommendations to avoid this type of bias would be the use of more objective measurements such as movement sensors or accelerometers or the use of a diary for measuring dietary intake.

Dichotomization of the behavior variables might be another limitation in this study, reducing the sensitivity of the data. Furthermore, use of BMI SDS scores in a longitudinal study instead of raw BMI scores is possibly a drawback because the within-subject variability in BMI values over time is much smaller for children at the end of the distribution, meaning that standardized scores are less sensitive to changes in overweight/obese and underweight children. At the follow up in 2012, children were involved up to 12.9 years, meaning that pubertal status could possibly have influenced our outcomes. Unfortunately, data on pubertal status was not available in our sample.

\section{Conclusions}

Comprehension of heterogeneity in the development of BMI and associations with modifiable health related behaviors is interesting for etiology and prevention, and may be helpful to identify high risk behaviors in high-risk groups and tailor interventions for children who are at higher risk. Our results indicate the importance of healthy lifestyle behaviors at a young age, and as confirmed in other studies investigating developmental trajectories in childhood, indicate that maternal BMI is a very important risk factor for the development of childhood overweight [31, 33]. Tailoring prevention activities to target high risk behaviors in early childhood, especially in low SES children, children of non-western ethnicity and children with an overweight mother is needed. The results can be used in practice such as in child health care services to identify and help children at risk for developing overweight and their parents by monitoring their dietary, physical activity and sedentary behaviors.

Although we did not find statistically significant results for the associations between the trajectories and health related behaviors, except for the participation in organized sports at follow-up, we have gained insight in the relationships between developmental patterns of BMI and health related behaviors, providing opportunities for further research regarding this subject.

\section{Additional files}

Additional file 1: Table S1. Characteristics of the study population based on the three-class model. Table S2. Health behaviors in the BMI SDS trajectories: the three-class model (DOC $95 \mathrm{~kb}$ )

Additional file 2: Figure S1. Three class linear model of BMI SDS trajectories. The latent growth patterns of BMI SDS are represented by mean trajectories of BMI standard deviation scores (SDS) (y-axis) at the different mean ages (x-axis). (JPG $54 \mathrm{~kb}$ )

\section{Abbreviations}

BIC: Bayesian information criterion; BLRT: Bootstrapped likelihood ratio test; BMI: Body mass index; Cl: Confidence interval; LCGMM: Latent class growth (mixture) modelling; OR: Odds ratios; SDS: Standard deviation score; SES: Socio-economic status

\section{Acknowledgements}

We would like to thank our partners in this monitoring study: the Zwolle city council, the local health services and several welfare organizations in the city. We would also like to thank the schools, children and parents who participated in this study, and acknowledge the help of all the students who performed the measurements in 2006, 2009 and 2012.

\section{Funding}

We received no specific grant from any funding agency in public, commercial or non-profit sectors. This study was funded by Windesheim University of Applied Sciences, the VU University and the local health services in Zwolle.

\section{Availability of data and materials}

The data that support the findings of this study are available on reasonable request from the corresponding author [MK]. The data are not publicly available due to agreements we have made concerning the exchange and use of our data.

\section{Authors' contributions}

JCS, CMR and TLSV were responsible for the study design. JCS and CMR supervised the data collection. MK was responsible for the statistical analyses and interpretation of the data in agreement with TH, JCS, CMR, EdJ and TLSV. MK wrote the first version of the manuscript and all authors participated in the revisions of the manuscript. All authors read and approved the final manuscript.

\section{Competing interests}

The authors declare that they have no competing interests.

\section{Consent for publication}

Not applicable.

\section{Ethics approval and consent to participate}

All procedures performed were in accordance with the ethical standards of the Medical Ethics Committee of the VU University Medical Centre in Amsterdam and with the WMA declaration of Helsinki. Informed consent was obtained from all parents of individual participants who were included in the study.

\section{Author details}

${ }^{1}$ Research Centre Healthy Cities, Knowledge Centre for Health and Social work, Windesheim University of Applied Sciences, PO box 10090, 8000 GB Zwolle, The Netherlands. ${ }^{2}$ Department of Health Sciences and the EMGO Institute for Health and Care Research, VU University Amsterdam, Amsterdam, The Netherlands. ${ }^{3}$ Department of Epidemiology and Biostatistics, VU University Medical Centre, Amsterdam, The Netherlands. ${ }^{4}$ Pedagogical Studies, Department for Health and Social Work, Windesheim University of Applied Sciences, Zwolle, The Netherlands.

Received: 28 April 2016 Accepted: 8 October 2016

Published online: 28 October 2016

\section{References}

1. Ogden $\mathrm{CL}$, et al. Prevalence of obesity and trends in body mass index among US children and adolescents, 1999-2010. JAMA. 2012;307(5):483-90.

2. Gupta N, et al. Childhood obesity in developing countries: epidemiology, determinants, and prevention. Endocr Rev. 2012;33(1):48-70. 
3. de Onis M, Blossner M, Borghi E. Global prevalence and trends of overweight and obesity among preschool children. Am J Clin Nutr. 2010;92(5):1257-64.

4. Wang $Y$, Lobstein T. Worldwide trends in childhood overweight and obesity. Int J Pediatr Obes. 2006;1(1):11-25.

5. Lobstein T, et al. Child and adolescent obesity: part of a bigger picture. Lancet. 2015;385(9986):2510-20.

6. Schokker DF, et al. Prevalence of overweight and obesity in the Netherlands. Obes Rev. 2007:8(2):101-7.

7. Schonbeck Y, et al. Increase in prevalence of overweight in Dutch children and adolescents: a comparison of nationwide growth studies in 1980, 1997 and 2009. PLoS One. 2011;6(11):e27608.

8. van den Hurk K, et al. Prevalence of overweight and obesity in the Netherlands in 2003 compared to 1980 and 1997. Arch Dis Child. 2007; 92(11):992-5.

9. Freedman DS, et al. The relation of childhood BMI to adult adiposity: the Bogalusa Heart Study. Pediatrics. 2005;115(1):22-7.

10. Magarey AM, et al. Predicting obesity in early adulthood from childhood and parental obesity. Int J Obes Relat Metab Disord. 2003;27(4):505-13.

11. Singh AS, et al. Tracking of childhood overweight into adulthood: a systematic review of the literature. Obes Rev. 2008;9(5):474-88.

12. Dietz WH. Health consequences of obesity in youth: childhood predictors of adult disease. Pediatrics. 1998;101(3 Pt 2):518-25.

13. Sun SS, et al. Childhood obesity predicts adult metabolic syndrome: the Fels Longitudinal Study. J Pediatr. 2008;152(2):191-200.

14. Daniels SR. Complications of obesity in children and adolescents. Int J Obes (Lond). 2009;33 Suppl 1:S60-5.

15. Must A, Strauss RS. Risks and consequences of childhood and adolescent obesity. Int J Obes Relat Metab Disord. 1999;23 Suppl 2:S2-11.

16. Han JC, Lawlor DA, Kimm SY. Childhood obesity. Lancet. 2010;375(9727):1737-48.

17. Popkin BM, Adair LS, Ng SW. Global nutrition transition and the pandemic of obesity in developing countries. Nutr Rev. 2012;70(1):3-21.

18. Biddle SJ, et al. Tracking of sedentary behaviours of young people: a systematic review. Prev Med. 2010;51(5):345-51.

19. Craigie AM, et al. Tracking of obesity-related behaviours from childhood to adulthood: A systematic review. Maturitas. 2011;70(3):266-84.

20. Telama R. Tracking of physical activity from childhood to adulthood: a review. Obes Facts. 2009;2(3):187-95.

21. Muthen B, Muthen LK. Integrating person-centered and variable-centered analyses: growth mixture modeling with latent trajectory classes. Alcohol Clin Exp Res. 2000;24(6):882-91.

22. Nagin DS. Analyzing developmental trajectories: A semiparametric, groupbased approach. Psychol Methods. 1999;4(2):139-57.

23. Nagin DS, Tremblay RE. Analyzing developmental trajectories of distinct but related behaviors: A group-based method. Psychol Methods. 2001;6(1):18-34

24. Muthen B. In: Kaplan D, editor. Latent variable analysis: growth mixture modelling and related techniques for longitudinal data. Newbury Park: Sage Publications; 2004.

25. Muthen B. The potential of growth mixture modelling. Infant Child Dev. 2006;15(6):623-5.

26. Jung T, Wickrama KAS. An introduction to latent class growth analysis and growth mixture modelling. Soc Personal Psychol Compass. 2008;2:302-17.

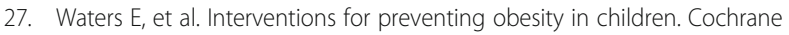
Database Syst Rev. 2011:12:CD001871.

28. Ventura AK, Loken E, Birch LL. Developmental trajectories of girls' BMI across childhood and adolescence. Obesity (Silver Spring). 2009;17(11):2067-74.

29. Magee CA, Caputi P, Iverson DC. Identification of distinct body mass index trajectories in Australian children. Pediatr Obes. 2013;8(3):189-98.

30. Haga C, et al. Developmental trajectories of body mass index among Japanese children and impact of maternal factors during pregnancy. PLoS One. 2012; 7(12):e51896.

31. Pryor LE, et al. Developmental trajectories of body mass index in early childhood and their risk factors: an 8-year longitudinal study. Arch Pediatr Adolesc Med. 2011;165(10):906-12.

32. Ziyab AH, et al. Developmental trajectories of Body Mass Index from infancy to 18 years of age: prenatal determinants and health consequences. J Epidemiol Community Health. 2014;68:934-41.

33. Li C, et al. Developmental trajectories of overweight during childhood: role of early life factors. Obesity (Silver Spring). 2007;15(3):760-71.

34. Magee CA, Caputi P, Iverson DC. The longitudinal relationship between sleep duration and body mass index in children: a growth mixture modeling approach. J Dev Behav Pediatr. 2013;34(3):165-73.
35. Hoekstra T, Barbosa_leiker C, Koppes LLJ, Twisk J. Developmental trajectories of body mass index throughout the life course: an application of latent class growth (mixture) modelling. Longit Life Course Stud. 2011;2(3):319-30.

36. Carter MA, et al. Trajectories of childhood weight gain: the relative importance of local environment versus individual social and early life factors. PLoS One. 2012;7(10):e47065.

37. Mustillo $\mathrm{S}$, et al. Obesity and psychiatric disorder: developmental trajectories Pediatrics. 2003;111(4 Pt 1):851-9.

38. Smith AJ, et al. Trajectories of childhood body mass index are associated with adolescent sagittal standing posture. Int J Pediatr Obes. 2011;6(2-2):e97-106.

39. Huang DY, Lanza HI, Anglin MD. Trajectory of adolescent obesity: exploring the impact of prenatal to childhood experiences. J Child Fam Stud. 2014; 23(6):1090-101.

40. Fredriks AM, et al. Body index measurements in 1996-7 compared with 1980. Arch Dis Child. 2000;82(2):107-12.

41. Cole TJ, et al. Establishing a standard definition for child overweight and obesity worldwide: international survey. BMJ. 2000;320(7244):1240-3.

42. Cole TJ, et al. Body mass index cut offs to define thinness in children and adolescents: international survey. BMJ. 2007;335(7612):194.

43. Singh AS, et al. Design of the Dutch Obesity Intervention in Teenagers (NRG-DOiT): systematic development, implementation and evaluation of a school-based intervention aimed at the prevention of excessive weight gain in adolescents. BMC Public Health. 2006;6:304

44. De Vries S, Bakker I, van Overbeek K, de Boer N, Hopman-Rock M. Kinderen in prioriteitswijken: lichamelijke (in)activiteit en overgewicht (Children in priority-neighbourhoods: physical (in)actvity and overweight). Leiden: TNO, Quality of Life; 2005

45. de Jong $E$, et al. Behavioural and socio-demographic characteristics of Dutch neighbourhoods with high prevalence of childhood obesity. Int J Pediatr Obes. 2011;6(3-4):298-305.

46. Voedingscentrum, Gezond Eten Gezond bewegen met kinderen 4-8 jaar, (Healthy Eating Healthy Physical Acivity with children aged 4-8 years). The Hague, the Netherlands: Dutch Nutrition Centre (Voedingscentrum); 2006.

47. Voedingscentrum, Gezond Eten Gezond bewegen met kinderen 9-12 jaar (Healthy Eating Healthy Physical Acivity with children aged 9-12 years). The Hague, the Netherlands: Dutch Nutrition Centre (Voedingscentrum); 2006.

48. de Jong E, et al. Association between TV viewing, computer use and overweight, determinants and competing activities of screen time in 4- to 13-year-old children. Int J Obes (Lond). 2013;37(1):47-53.

49. Kemper HCG, Ooijendijk WTM, Stiggelbout M. Consensus over de Nederlandse norm voor gezond bewegen (Consensus on the Dutch standard for healthy exercise). TSG. 2000;78(3):180-3.

50. Muthen L, Muthen B. Mplus user's guide. 5th ed. Los Angeles: Muthen \& Muthen; 2010.

51. Muthen B, Muthen L, Mplus 6.12 (Computer program). 2011.

52. Nylund $\mathrm{KL}$, Asparoutiov T, Muthen BO. Deciding on the number of classes in latent class analysis and growth mixture modeling: A Monte Carlo simulation study. Struc Equa Modeling Multdiscip J. 2007;14(4):535-69.

53. Schwarz G. Estimating the dimension of a model. Ann Stat. 1978;6:461-4.

54. Raftery AE. Bayesian model selection in social research. Soc Methodol. 1995. 25:111-63.

55. McLachlan G, Peel D. Finite mixture models. New York: Wiley; 2000

56. Connell AM, Frye AA. Growth mixture modelling in developmental psychology: Overview and demonstration of heterogeneity in developmental trajectories of adolescent antisocial behaviour. Infant Child Dev. 2006;15(6):609-21.

57. Twisk J, Hoekstra T. Classifying developmental trajectories over time should be done with great caution: a comparison between methods. J Clin Epidemiol. 2012:65(10):1078-87.

58. Jansen PW, et al. Family and neighbourhood socioeconomic inequalities in childhood trajectories of BMI and overweight: Iongitudinal study of Australian children. PLoS One. 2013;8(7):e69676.

59. de Bourdeaudhuij I. Personal and family determinants of dietary behaviour in adolescents and their parents. Psychol Health. 2000;15:751-70. 